\title{
INTERAÇÃO INTERINSTITUCIONAL: O CASO DO PROGRAMA DE DESENVOLVIMENTO DA EDUCAÇÃO (PDE) / UNIVERSIDADE FEDERAL DO PARANÁ (UFPR) E O DESENVOLVIMENTO REGIONAL
}

\author{
Inter-institutional interaction: the case of the Education Development \\ Programme/Universidade Federal do Paraná (UFPR) and regional development
}

Marlene Ferreira Gomes Mortagua Walflor ${ }^{1}$

Orlando Cesar Devai

Geraldine Marie Rita Vieira ${ }^{3}$

\begin{abstract}
RESUMO
O Programa de Desenvolvimento da Educação (PDE) implementado pela UFPR, pela Secretaria de Estado da Educação do Paraná e pela Secretaria de Ciência, Tecnologia e Ensino Superior do Paraná, é um exemplo das interações necessárias para assegurar a efetividade e a sustentabilidade da extensão universitária. Objetiva oferecer subsídios para um trabalho de formação continuada com reflexão crítica sobre as práticas e de (re)construção permanente e coletiva das identidades profissionais. Metodologicamente, o PDE dá ênfase no aprofundamento teórico, na integração teórico-prática, na troca de experiências, na produção de materiais didáticos e na interlocução entre professores das escolas da rede de ensino estadual básico e da universidade. Esse modelo de formação continuada, que ocorre durante dois anos, proporciona ao professor retorno às atividades acadêmicas de sua área de formação inicial. O Programa é estruturado em três grandes eixos: atividades de integração teórico-práticas, atividades de aprofundamento teórico e atividades didático-pedagógicas com suporte tecnológico. Os principais resultados: três turmas formadas com projetos de intervenção na escola, já implantados em escolas do ensino básico localizadas em Curitiba, União da Vitória e municípios das Regiões Metropolitanas Norte e Sul de Curitiba, e do Litoral Norte e Sul do Paraná. Conclusão: A universidade apropriou-se de uma metodologia para a formação continuada, ampliou sua rede de aliança e pretende consolidar a relação Universidade com a Escola e facilitar o diálogo com os agentes educacionais da região.
\end{abstract}

Palavras-chave: interação interinstitucional; educação básica; formação continuada.

\begin{abstract}
The Education Development Program (Programa de Desenvolvimento da Educação PDE) implemented by UFPR, the Paraná State Department of Education and the Paraná Science, Technology and Higher Education Secretariat is one example of the

\footnotetext{
1 Doutora, UFPR, trabalhou na PROEC/Coordenadoria de Extensão. E-mail: marlene.mortagua@gmail.com

2 Doutorado em Desenvolvimento Econômico. UFPR, PROPLAN. E-mail: devai@ufpr.br.

3 Especialista, UFPR, trabalhou junto à PROEC/Coordenadoria de Extensão - PDE. E-mail: geraldinevieira.ufpr@gmail.com
}

WALFLOR, Marlene F. G. M; DEVAI, Orlando C.; VIEIRA, Geraldine M.R. Interação interinstitucional: o caso do Programa de Desenvolvimento da Educação (PDE) / Universidade Federal do Paraná (UFPR) e o desenvolvimento regional. Extensão em Foco, Curitiba: Editora da UFPR, nr.9, jan/jun 2014, p.61-78. ISSN 2358-7180. 
necessary interactions to ensure effectiveness and sustainability to university extension. It aims to offer a subsidy for regular job training with critical reflection on practices and permanent and collective (re)construction of professional identities. Methodologically, the PDE emphasizes more theoretical studies on integrating theory and practice, the exchange of experiences, the production of teaching materials and communication among teachers from elementary state schools and from the university. This model of continuous training, which is established during two years, provides the teachers' return to their academic activities taking their degree area. The program is structured in three main fields: theoretical and practical integration activities, theory deepening practices and didactic and pedagogical activities supported by technology. Main results: three groups formed with the school intervention projects already implemented in primary schools located in Curitiba, União da Vitória and other municipalities from Northern and Southern Metropolitan Areas of Curitiba, and from the Northern and Southern Coast of Paraná. Conclusion: The university has taken a methodology for continuing education, it has expanded its alliance network and it aims to consolidate the relationship between the University and Schools and facilitate the dialogue with the educational agents in the region.

Keywords: inter-institutional interaction; elementary schooling; continuous training.

\section{RESÚMEN}

El Programa de Desarrollo de la Educación (PDE), ejecutado por la UFPR, el Secretaría de Educación del Estado de Paraná y la Secretaría de Ciencia, Tecnología y Enseñanza Superior de Paraná, es un ejemplo de las interacciones necesarias para garantizar la eficacia y la sostenibilidad de la extensión universitaria. Tiene como objetivo ofrecer subsidios para un trabajo de la formación continua con reflexión crítica sobre las prácticas y la (re)construcción permanente y colectiva de identidades profesionales. Metodológicamente, el PDE enfatiza el estudio teórico, la integración de la teoría y la práctica, el intercambio de experiencias, la producción de materiales de enseñanza y la comunicación entre los profesores de las escuelas de enseñanza básica estatal y de la universidad. Este modelo de formación continua, que se produce durante dos años, proporciona al profesor retorno a las actividades académicas de su área de formación inicial. El Programa es estructurado en tres áreas principales: actividades de integración teórica y actividades prácticas, actividades de profundización teórica y actividades didácticas y pedagógicas con el apoyo de la tecnología. Los principales resultados: tres grupos formados con proyectos de intervención escolar, que ya se aplican en las escuelas primarias ubicadas en Curitiba, União da Vitória municipios de las Regiones Norte y

Sur del Área Metropolitana de Curitiba, y del Norte y Sur de la Costa del Paraná. Conclusión: La universidad ha tomado una metodología para la formación continua, ha ampliado su red de alianzas y desea consolidar la relación de la Universidad con la escuela y facilitar el diálogo con los agentes educativos en la región.

Palabras-clave: interacción interinstitucional; educación básica; educación continua

\section{Introdução}

As ações de extensão, constituintes essenciais da formação, concretizam o princípio que fundamenta a educação nacional. A educação abrange os processos formativos que se desenvolvem na vida familiar, na WALFLOR, Marlene F. G. M; DEVAI, Orlando C.; VIEIRA, Geraldine M.R. Interação interinstitucional: o caso do Programa de Desenvolvimento da Educação (PDE) / Universidade Federal do Paraná (UFPR) e o desenvolvimento regional. Extensão em Foco, Curitiba: Editora da UFPR, nr.9, jan/jun 2014, p.61-78. ISSN 2358-7180. 
convivência humana, no trabalho, nas instituições de ensino e pesquisa, nos movimentos sociais, nas organizações da sociedade civil e nas manifestações culturais. Art. 1ํ Lei de Diretrizes e Bases da Educação Nacional. 1996.

As ações de extensão devem ser formuladas e implementadas de forma articulada com outras instâncias que também são fonte do saber.

A universidade possui um rico capital humano e social. Congrega cientistas, técnicos e artistas, em atividades de estudo e pesquisa capazes de oferecer valiosos subsídios para a sociedade. A integração de esforços dos diversos setores da sociedade no processo de desenvolvimento local/regional traz como consequência a racionalização de recursos institucionais (humanos, físicos, materiais e financeiros) envolvidos, otimizando os resultados. Este é o caso da interação da Universidade Federal do Paraná (UFPR), com o Governo do Estado do Paraná para realizar o Programa de Desenvolvimento Educacional - PDE/PR.

A Secretaria de Estado da Educação do Paraná (SEED), em parceria com a Secretaria de Estado da Ciência, Tecnologia e Ensino Superior (SETI), instituiu o PDE/PR, como política educacional inovadora de Formação Continuada de professores da rede pública estadual. O Programa articula-se ao Plano de Carreira dos professores do Quadro Próprio do Magistério (QPM), conforme a Lei Complementar no 103/04.

Considerando o seu caráter interinstitucional, o Programa é coordenado pela a SEED e a SETI. A primeira envolvendo 2.139 escolas de Educação Básica da rede pública estadual (aproximadamente 61 mil professores QPM) e 32 Núcleos Regionais de Educação; a segunda envolvendo as 12(doze) Instituições de Ensino Superior Estaduais e as duas Instituições de Ensino Superior presentes no Estado, a partir de Convênio Institucional.

O Programa propõe um conjunto de atividades organicamente articuladas, definidas a partir das necessidades da Educação Básica, buscando no Ensino Superior a contribuição solidária e compatível com o nível de qualidade desejado para a educação pública no Estado do Paraná.

Consubstanciada nesses posicionamentos é que a UFPR estabeleceu interação com as SEED e com a SETI para realizar o PDE/PR como atividade

WALFLOR, Marlene F. G. M; DEVAI, Orlando C.; VIEIRA, Geraldine M.R. Interação interinstitucional: o caso do Programa de Desenvolvimento da Educação (PDE) / Universidade Federal do Paraná (UFPR) e o desenvolvimento regional. Extensão em Foco, Curitiba: Editora da UFPR, nr.9, jan/jun 2014, p.61-78. ISSN 2358-7180. 
pedagógica que reconhece a singularidade das instituições e de seus profissionais que se apresentam com uma dinâmica própria que deve ser considerada nos processos de intervenção educacional.

\section{Contextualização}

Revendo a discussão em torno do papel da universidade para com as demandas educativas do meio local, é que se tem repensado as ações de extensão universitária. A universidade não só se estende, se vincula ou se projeta a outros setores da sociedade a qual pertence, em que propicia um diálogo de saberes, entre instituições existentes no entorno, com outros saberes populares que interagem com os saberes científicos, com o qual a universidade também aprende e capta insumos para atualizar-se em suas orientações acadêmicas, como produto de contato com realidades sociais e de diferentes atores. Nessa interação com outros setores da sociedade, a universidade deve apresentar-se devidamente articulada em suas três dimensões básicas: formação, pesquisa e extensão, aspectos bastante complexos, dadas as formas como estão organizadas academicamente e administrativamente a maioria de nossas universidades, como podemos dizer, segundo Gibbons (1998), são universidades MODO1. Segundo Axel Dichiksson, esta "é uma instituição ativa, dinâmica e social, baseada na formação de trabalhadores do conhecimento com um compromisso de alto nível e responsabilidade pela mudança social, democracia, paz e desenvolvimento de forma sustentável” (2006, p.12). É uma universidade de qualidade em que o valor das produções sociais e da transferência de conhecimento é apresentado como um princípio organizacional, o eixo de suas mudanças reside na natureza de seus processos educativos, e o perfil da instituição responde aos desafios colocados pela transição democrática e o desenvolvimento para o bem-estar.

Tampouco, pode-se desconhecer a pergunta relacionada com a extensão, sobre como a universidade contribui ao desenvolvimento regional e de que maneira sua atuação está ligada a da sociedade civil, que interatua com a sociedade política e a sociedade econômica; cada uma dentro de certas

WALFLOR, Marlene F. G. M; DEVAI, Orlando C.; VIEIRA, Geraldine M.R. Interação interinstitucional: o caso do Programa de Desenvolvimento da Educação (PDE) / Universidade Federal do Paraná (UFPR) e o desenvolvimento regional. Extensão em Foco, Curitiba: Editora da UFPR, nr.9, jan/jun 2014, p.61-78. ISSN 2358-7180. 
ênfases em suas racionalidades como: o ético para a primeira, e o pragmático e técnico para as outras duas. Luís Bacigalupo (2009) explica que a interação das três sociedades com suas racionalidades próprias são as que geram desenvolvimento entendido em termos amplos, destacando como a sociedade civil e a universidade, dentro de sua racionalidade ética, devem permanentemente estar repreendendo eticamente as outras duas e propiciando que haja uma maior densidade institucional em uma região para conseguir desenvolvimento.

Assim, a extensão universitária pode ser entendida como uma dimensão essencial da universidade, que está intimamente integrada com a formação e com a pesquisa, que the permite interagir com outros setores da sociedade, sem perder sua identidade, para contribuir na solução de problemas sociais de seu entorno, assim como aprender a levar essa aprendizagem para sua transformação interna nas dimensões substantivas e em sua organização e gestão como entidade do conhecimento. Também permite a universidade em meio de sua interdependência, fortalecer sua identidade e ter mais clara a "ideia de universidade" a partir das próprias realidades sociais. Desta forma a extensão é uma tarefa acadêmica da universidade e visa estabelecer processos de contínua interação e integração com o social, meio ambiente e comunidade, bem como outras tarefas substantivas e está sujeita à lógica dos ciclos de longa acumulação/renovação. Diante da possibilidade de bons sistemas de informação, a universidade com claras políticas de extensão, pode contribuir de maneira mais eficaz na solução de problemáticas sociais específicas e fazer que essas comunidades cada vez mais passem a possuir uma capacidade de autogestão para seus próprios problemas. É no fundo uma maneira de transferir conhecimento acadêmico útil em função de demandas sociais concretas em que participam os estudantes, os professores, os pesquisadores e a própria instituição educativa de maneira integral, interagindo com outros atores sociais.

Essa atuação integral deve ter medidas concretas em torno da problemática social que se está atendendo, sem desconhecer uma série de externalidades que deixam uns capitais intangíveis úteis para a universidade,

WALFLOR, Marlene F. G. M; DEVAI, Orlando C.; VIEIRA, Geraldine M.R. Interação interinstitucional: o caso do Programa de Desenvolvimento da Educação (PDE) / Universidade Federal do Paraná (UFPR) e o desenvolvimento regional. Extensão em Foco, Curitiba: Editora da UFPR, nr.9, jan/jun 2014, p.61-78. ISSN 2358-7180. 
tais como: melhoramento da formação integral e as competências de cidadania para os membros da comunidade universitária que participam; melhoramento na imagem institucional e do grau de legitimação frente à sociedade; aprendizagens sociais que permitem replanejar os currículos, as líneas e metodologias de pesquisa, os métodos de trabalho com comunidades; valorização de novas pedagogias e didáticas de ordem social que enriquecem os professores em seu trabalho formativo; aumento da autoestima institucional que permite adquirir e renovar novos compromissos com o social como parte de sua missão institucional; ensinos sobre medição de impactos; determinação da evolução de fatores que estimulam a inovação e o empreendimento social como características de uma universidade contemporânea; possibilidades de novas alianças com múltiplos atores sociais; desenvolvimento de novas possibilidades institucionais a partir de intervenções complexas com o trabalho interdisciplinar e interinstitucional.

\section{O Programa de Desenvolvimento Educacional - PDE/PR e PDE/UFPR}

O aspecto central do Programa é a previsão de tempo livre para estudos, demonstrando a preocupação com a qualidade da formação continuada de professores da rede de ensino. Assim, tem a duração de dois anos, sendo que no primeiro ano o professor PDE afasta-se de suas atividades em $100 \%$ e no segundo, em $25 \%$, momento em que implementa o projeto de Intervenção Pedagógica na escola de origem.

Esse novo modelo de Formação Continuada proporciona ao professor o retorno às atividades acadêmicas de sua área de formação inicial. Realizado, de forma presencial nas universidades públicas do estado do Paraná, incorpora também em sua organização curricular a atividade a distância denominada Grupo de Trabalho em Rede - GTR - por meio do qual o professor PDE socializa aos demais professores da rede pública estadual de educação básica os resultados de seus estudos e produções elaboradas no âmbito do programa.

A implementação do Programa PDE/PR na Universidade Federal do Paraná se efetiva por meio do programa de extensão universitária denominado "Programa de Desenvolvimento da Educação - PDE/UFPR", que objetiva

WALFLOR, Marlene F. G. M; DEVAI, Orlando C.; VIEIRA, Geraldine M.R. Interação interinstitucional: o caso do Programa de Desenvolvimento da Educação (PDE) / Universidade Federal do Paraná (UFPR) e o desenvolvimento regional. Extensão em Foco, Curitiba: Editora da UFPR, nr.9, jan/jun 2014, p.61-78. ISSN 2358-7180. 
oportunizar a formação continuada de professores ligada às reais necessidades de enfrentamento de problemas presentes na Educação Básica, criando condições efetivas de debate e promoção de espaços para a construção coletiva do saber, através de cursos, seminários, palestras, encontros de orientação e estudos correlatos. As atividades do objeto abrangem os conteúdos indicados na Proposta Político Pedagógica do PDE, tendo como referência as Diretrizes Curriculares da Rede Pública Estadual de Educação Básica.

A estrutura organizacional do Programa de Desenvolvimento Educacional está representada, para fins didáticos, no Plano Integrado de Formação Continuada, o qual constitui-se de três grandes eixos de atividades, quais sejam: atividades de integração teórico-práticas, atividades de aprofundamento teórico e atividades didático-pedagógicas com utilização de suporte tecnológico. Essas atividades são realizadas no decorrer do programa, composto de quatro períodos semestrais, distribuídos em dois anos, inclusive para os professores titulados, os quais podem solicitar aproveitamento parcial de sua titulação. Essa organização não pode ser considerada de forma estanque, uma vez que o pressuposto é de que os conteúdos das atividades que compõe os eixos integrem-se e articulem-se de tal modo que as categorias que identificam cada um dos eixos estejam presentes em todas as atividades do Programa.

O primeiro eixo prioriza as atividades voltadas para a integração teóricoprática, enquanto parte da proposta de formação continuada do PDE, estando nele contemplado: o projeto de intervenção pedagógica na escola, o processo de orientação nas IES, a produção didático-pedagógica direcionada para a implementação do projeto na escola, a coordenação de grupo de apoio à implementação do projeto de intervenção pedagógica e um artigo científico, considerado como trabalho de conclusão do programa.

As atividades desse primeiro eixo são desenvolvidas com orientação dos professores orientadores das instituições de ensino superior do estado, a partir da definição do objeto de estudo pelo professor PDE, de acordo com sua área/disciplina de ingresso no programa.

WALFLOR, Marlene F. G. M; DEVAI, Orlando C.; VIEIRA, Geraldine M.R. Interação interinstitucional: o caso do Programa de Desenvolvimento da Educação (PDE) / Universidade Federal do Paraná (UFPR) e o desenvolvimento regional. Extensão em Foco, Curitiba: Editora da UFPR, nr.9, jan/jun 2014, p.61-78. ISSN 2358-7180. 
O segundo eixo, atividades de aprofundamento teórico, identifica-se com a própria natureza das atividades propostas e contribui para o aprofundamento teórico das questões educacionais em geral e das questões específicas do currículo da educação básica da rede estadual em específico. Nesse eixo, o professor PDE participa de um conjunto de atividades ofertadas pelas IES conveniadas e pelo PDE/SEED, objetivando ampliar, aprofundar e atualizar os seus conhecimentos, sendo que seu conteúdo abrange temas relativos aos fundamentos da educação, à metodologia científica e aos conteúdos curriculares específicos de sua área de ingresso no PDE, possibilitando aprofundamento teórico metodológico, dentre outras possibilidades de oferta. As atividades desenvolvidas nesse eixo compreendem: cursos, seminários, encontros de área, eventos de inserção acadêmica e teleconferências.

O terceiro eixo, atividades didático-pedagógicas com utilização de suporte tecnológico, possui uma identidade específica na sua relação com a formação tecnológica para possibilitar a interação do professor PDE com os demais professores da Rede, através de Grupos de Trabalho em Rede - GTR, com utilização da plataforma MOODLE.

\section{Material e Métodos}

Para realizar o estudo foi adotada a pesquisa descritiva e como instrumento o Marco Lógico e consulta de documentos (propostas do PDE/UFPR, relatórios do PDE/UFPR, propostas e relatórios de Cursos de Extensão, ficha de acompanhamento do professor PDE pelo professor orientador); o banco de dados SACIR/PDE; questionários ao coordenador do PDE/UFPR, ao professor orientador de professor PDE e ao professor PDE; entrevista; observação.

O estudo ocorreu em três momentos: na concepção com a elaboração do Marco Lógico, na execução (processo), na constatação de resultados e impactos.

O Marco Lógico é uma análise que tem por base caracterizar a situação inicial do programa. Este foi elaborado a partir da proposta do Programa de Extensão Universitária - PDE/UFPR aprovada para o período de 2007/2008 e

WALFLOR, Marlene F. G. M; DEVAI, Orlando C.; VIEIRA, Geraldine M.R. Interação interinstitucional: o caso do Programa de Desenvolvimento da Educação (PDE) / Universidade Federal do Paraná (UFPR) e o desenvolvimento regional. Extensão em Foco, Curitiba: Editora da UFPR, nr.9, jan/jun 2014, p.61-78. ISSN 2358-7180. 
das propostas de continuidade do mesmo para os períodos 2008/2009 e 2009/2011, com a finalidade de ser um instrumento metodológico para a análise clara da relação de meios/fins das atividades que levam a produtos (ou componentes) requeridos para atingir o propósito estabelecido (objetivo principal do programa); a especificação precisa das atividades; a descrição de indicadores de desempenho e de suas fontes de verificação; a especificação dos pressupostos principais que poderiam condicionar o sucesso do programa e o marco de referência para indicar as experiências adquiridas e incorporá-las e outros programas/projetos.

A avaliação de processo objetiva verificar a realização das atividades relacionadas aos objetivos estabelecidos. Neste momento foram identificados os indicadores de progresso (quantitativos e qualitativos) das atividades e os meios de verificação das informações. Para tanto foram verificadas as propostas e relatórios: do PDE/UFPR; de cursos de extensão gerais e específicos programados para professores PDE; de projetos de pesquisa e de extensão com oferta de vagas para inserção do professor PDE; a oferta de vagas em disciplinas isoladas de cursos de graduação e pós-graduação para inserção de professores PDE; a oferta de vagas para professores PDE em grupos de estudos em diferentes áreas do conhecimento; convites para participação e relatórios de eventos de extensão (encontros, seminários, congressos, entre outros). As informações foram confirmadas mediante consulta a 552 fichas de acompanhamento do professor PDE e os comprovantes de participação e frequência nas diferentes atividades, correspondendo a totalidade dos professores concluintes do programa.

A avaliação de resultados refere-se à verificação dos métodos e procedimentos utilizados e deve denotar as transformações geradas pelas atividades. Neste momento foram identificados os indicadores quantitativos e qualitativos dos resultados, assim como os meios de verificação e o impacto refere-se à permanência ou sustentabilidade no tempo das transformações decorrentes das ações implementadas, ou seja, sua efetividade.

A verificação das informações da avaliação de resultados e de impactos foram viabilizadas pela aplicação de questionário composto por questões

WALFLOR, Marlene F. G. M; DEVAI, Orlando C.; VIEIRA, Geraldine M.R. Interação interinstitucional: o caso do Programa de Desenvolvimento da Educação (PDE) / Universidade Federal do Paraná (UFPR) e o desenvolvimento regional. Extensão em Foco, Curitiba: Editora da UFPR, nr.9, jan/jun 2014, p.61-78. ISSN 2358-7180. 
objetivas e discursivas direcionado aos professores PDE, professores orientadores e coordenadores do PDE/UFPR e pelo registro de entrevistas e observações realizadas em escolas da rede pública estadual localizadas no município de Curitiba, de sua região Metropolitana e do Litoral, onde atuam professores egressos das primeiras três turmas do programa PDE/UFPR.

\section{Resultados e Discussão}

O PDE/UFPR foi implantado como programa de extensão universitária em junho de 2007, e de sua avaliação pela SEED e SETI, no primeiro ano, decorreu sua continuidade como programa de extensão.

Assim sendo, até junho de 2011, 552 professores se inscreveram no PDE/UFPR.

TABELA 1 - PROGRAMA DE DESENVOLVIMENTO EDUCACIONAL - PDE/UFPR NÚMERO DE PROFESSORES ATENDIDOS/TURMA / ÁREA DE ATUAÇÃO

\begin{tabular}{|c|c|c|c|c|c|c|c|c|c|c|c|c|c|c|c|c|c|c|}
\hline $\begin{array}{l}\text { Área } \\
\text { Tur } \\
\text { ma }\end{array}$ & $\frac{\frac{\pi}{8}}{\frac{0}{0}}$ & 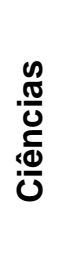 & 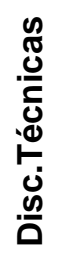 & 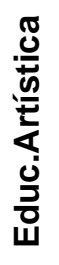 & 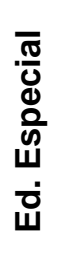 & 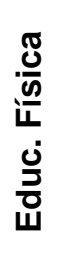 & 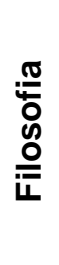 & $\frac{\mathbb{J}}{\frac{0}{6}}$ & $\begin{array}{l}\frac{\pi}{\pi} \\
\frac{\pi}{0} \\
\frac{0}{0} \\
\mathbb{U}\end{array}$ & 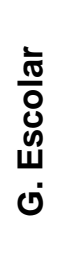 & 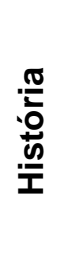 & 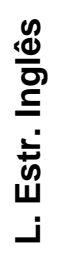 & 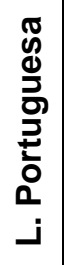 & 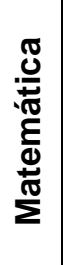 & $\begin{array}{l}\frac{\pi}{5} \\
\frac{0}{0} \\
\frac{\pi}{8} \\
0\end{array}$ & 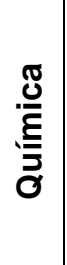 & $\begin{array}{l}\frac{\pi}{6} \\
\frac{0}{0} \\
\frac{0}{0} \\
\dot{\circ}\end{array}$ & $\begin{array}{l}\bar{\pi} \\
\text { 0 }\end{array}$ \\
\hline $\begin{array}{l}2007 \\
/ 08\end{array}$ & 6 & $\begin{array}{l}1 \\
2\end{array}$ & 1 & 1 & $\begin{array}{l}1 \\
3\end{array}$ & $\begin{array}{l}1 \\
4\end{array}$ & 1 & 1 & $\begin{array}{l}2 \\
1\end{array}$ & $\begin{array}{l}1 \\
4\end{array}$ & $\begin{array}{l}1 \\
9\end{array}$ & $\begin{array}{l}1 \\
6\end{array}$ & $\begin{array}{l}2 \\
5\end{array}$ & $\begin{array}{l}1 \\
1\end{array}$ & $\begin{array}{l}3 \\
4\end{array}$ & 3 & 0 & 192 \\
\hline $\begin{array}{l}2008 \\
/ 09\end{array}$ & 8 & 8 & 0 & $\begin{array}{l}1 \\
2\end{array}$ & $\begin{array}{l}1 \\
6\end{array}$ & $\begin{array}{l}2 \\
7\end{array}$ & 0 & 1 & 7 & $\begin{array}{l}1 \\
1\end{array}$ & $\begin{array}{l}3 \\
7\end{array}$ & $\begin{array}{l}1 \\
0\end{array}$ & 2 & \begin{tabular}{l|}
1 \\
0
\end{tabular} & $\begin{array}{l}3 \\
2\end{array}$ & 6 & 1 & 188 \\
\hline $\begin{array}{l}2009 \\
/ 11\end{array}$ & 1 & $\begin{array}{l}1 \\
3\end{array}$ & 0 & 0 & 9 & $\begin{array}{l}1 \\
9\end{array}$ & 2 & 0 & $\begin{array}{l}1 \\
4\end{array}$ & $\begin{array}{l}1 \\
0\end{array}$ & $\begin{array}{l}2 \\
8\end{array}$ & 8 & \begin{tabular}{l|}
2 \\
5
\end{tabular} & \begin{tabular}{l|}
1 \\
5
\end{tabular} & $\begin{array}{l}2 \\
6\end{array}$ & 2 & 0 & 172 \\
\hline Total & $\begin{array}{l}1 \\
5\end{array}$ & $\begin{array}{l}3 \\
3\end{array}$ & 1 & $\begin{array}{l}1 \\
3\end{array}$ & $\begin{array}{l}3 \\
8\end{array}$ & $\begin{array}{l}6 \\
0\end{array}$ & 3 & 2 & $\begin{array}{l}4 \\
2\end{array}$ & $\begin{array}{l}3 \\
5\end{array}$ & $\begin{array}{l}8 \\
4\end{array}$ & $\begin{array}{l}3 \\
4\end{array}$ & $\begin{array}{l}5 \\
2\end{array}$ & $\begin{array}{l}3 \\
6\end{array}$ & $\begin{array}{l}9 \\
2\end{array}$ & $\begin{array}{l}1 \\
1\end{array}$ & 1 & 552 \\
\hline
\end{tabular}

Fonte:PROEC/COEX/PDE(2011)

Participaram no PDE/UFPR, nesse período, além dos professores selecionados atuantes em escolas de diferentes municípios dessas regiões, profissionais dos Núcleos Regionais de Ensino (NRE) da região Metropolitana Sul, Metropolitana Norte, Curitiba, Paranaguá e União da Vitória.

WALFLOR, Marlene F. G. M; DEVAI, Orlando C.; VIEIRA, Geraldine M.R. Interação interinstitucional: o caso do Programa de Desenvolvimento da Educação (PDE) / Universidade Federal do Paraná (UFPR) e o desenvolvimento regional. Extensão em Foco, Curitiba: Editora da UFPR, nr.9, jan/jun 2014, p.61-78. ISSN 2358-7180. 
FIGURA 1 - MUNICÍPIOS DE ORIGEM DO PROFESSOR PDE/UFPR 2007/2011

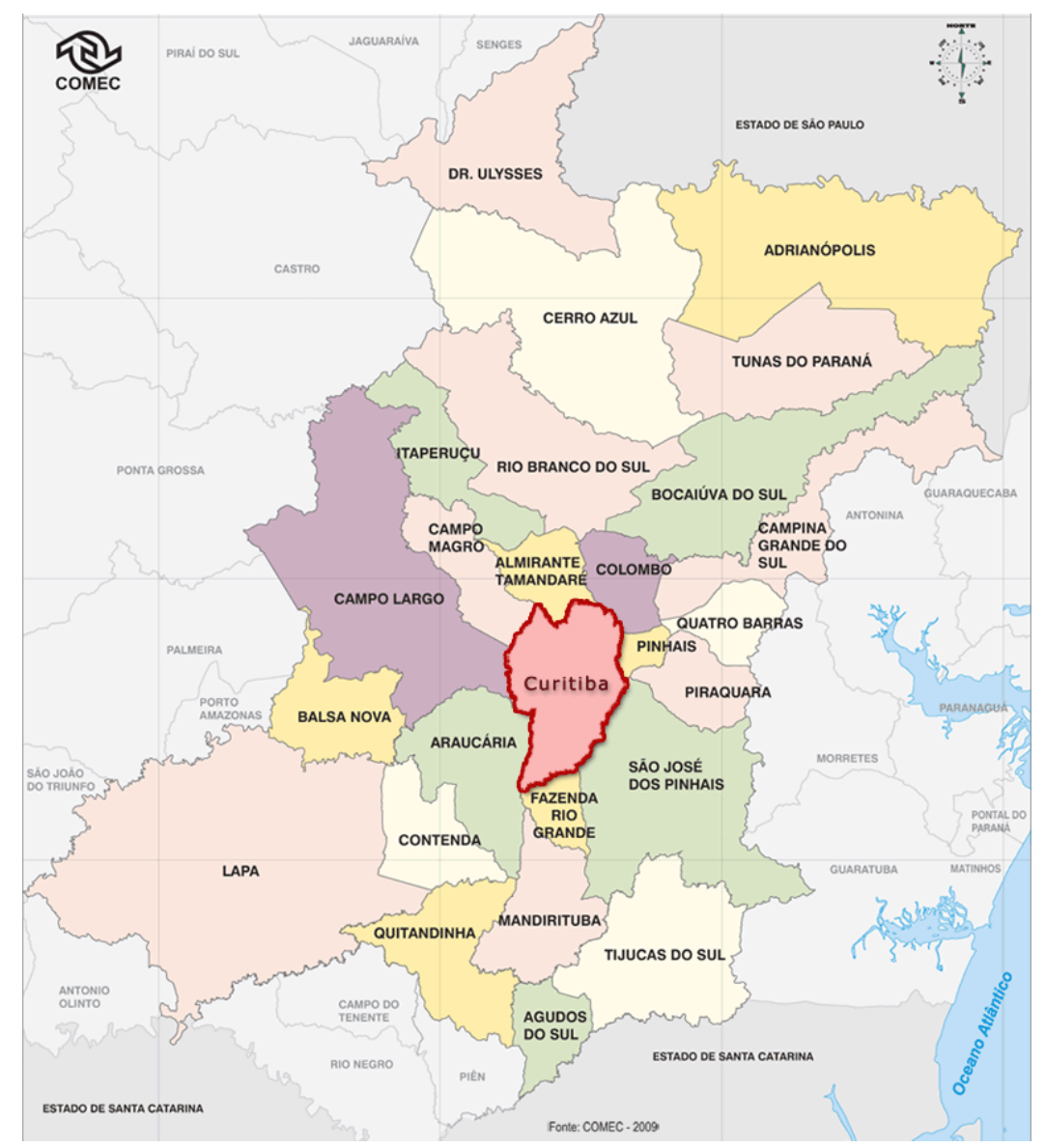

Fonte: <http://www.guiageoparana.com/rmc.htm>

Na primeira edição, 2007-2008, os 192 professores PDE inscritos, orientados por 61 professores vinculados a essas unidades de ensino, elaboraram e implantaram o Projeto de Intervenção Pedagógica na Escola (PIPE), produziram material didático e o artigo científico requerido para a conclusão do PDE/PR. Dos 192 concluintes, 167 são professores graduados e 25 titulados.

Na segunda edição, 2008 - 2009, os 188 professores PDE inscritos, orientados por 63 professores vinculados a essas unidades de ensino, elaboraram o Projeto de Intervenção Pedagógica na Escola, e 186 implantaram o PIPE, produziram material didático e o artigo científico requerido para a conclusão do PDE/PR. Dos 186 concluintes, 173 são professores graduados e 13 titulados. Os 02 (dois) não concluintes são graduados. Um desses concluiu WALFLOR, Marlene F. G. M; DEVAI, Orlando C.; VIEIRA, Geraldine M.R. Interação interinstitucional: o caso do Programa de Desenvolvimento da Educação (PDE) / Universidade Federal do Paraná (UFPR) e o desenvolvimento regional. Extensão em Foco, Curitiba: Editora da UFPR, nr.9, jan/jun 2014, p.61-78. ISSN 2358-7180. 
o Programa PDE/PR implantado o Projeto de Intervenção Pedagógica na Escola em 2010.

Na terceira edição, 2009 - 2011, os 172 professores PDE inscritos, orientados por 67 professores vinculados a essas unidades de ensino, elaboraram o Projeto de Intervenção Pedagógica na Escola, e 168 implantaram - PIPE, produziram material didático e o artigo científico requerido para a conclusão do PDE/PR. Dos 168 concluintes, 154 são professores graduados e 14 titulados. Os 04 (quatro) não concluintes são graduados.

Os 546 professores PDE concluintes do programa no período 2007 2011, além de elaborarem e implantarem o Projeto de Intervenção Pedagógica na Escola participaram de seminários de integração, encontros de área, cursos gerais e específicos organizados pela UFPR conforme previsto para a formação continuada e também de atividades de ensino, pesquisa e extensão, nas diferentes áreas do conhecimento programadas pelos setores e departamentos acadêmicos da UFPR.

Os 546 professores PDE concluintes participaram de três seminários de integração e de quatro encontros de área, programados para cada turma, registrando $100 \%$ de frequência.

Os 67 cursos, gerais e específicos organizados pela UFPR, receberam 3.158 inscrições de professores PDE. Destes 2.522 registraram frequência superior a $80 \%$.

Das atividades de ensino, pesquisa e extensão realizadas para a comunidade acadêmica, por diferentes departamentos da UFPR, 228 receberam professores PDE. Dos concluintes do programa no período, $21 \%$ participaram de atividades de ensino, $1 \%$ de pesquisa e $100 \%$ de atividades de extensão.

Dos professores PDE concluintes que responderam o questionário e entrevista $18.19 \%$ são da área de ciências, $18.19 \%$ de pedagogia, $18.19 \%$ de língua portuguesa, $13.63 \%$ de língua estrangeira moderna, $13.63 \%$ de educação física, 9.09\% de matemática, 4.54\% de gestão escolar e 4.54\% da área de história.

WALFLOR, Marlene F. G. M; DEVAI, Orlando C.; VIEIRA, Geraldine M.R. Interação interinstitucional: o caso do Programa de Desenvolvimento da Educação (PDE) / Universidade Federal do Paraná (UFPR) e o desenvolvimento regional. Extensão em Foco, Curitiba: Editora da UFPR, nr.9, jan/jun 2014, p.61-78. ISSN 2358-7180. 
Os professores PDE manifestaram que o programa é uma ação importante na formação continuada do professor e que os seminários, encontros e cursos ampliaram o conhecimento quanto às possibilidades de fundamentar e desenvolver práticas pedagógicas importantes para a valorização profissional e também a participação do professor nos cursos gerais e específicos tirou o professor PDE da sua rotina propiciando 0 aprofundamento do conhecimento nas diferentes áreas.

Manifestaram, também, que a implantação do projeto de intervenção pedagógica indiretamente constituiu-se em elemento para a apresentação de novas propostas de ação na escola e destacam nas experiências vivenciadas no decorrer da implantação do projeto a participação de diferentes profissionais atuantes na escola, o envolvimento de profissionais de serviços gerais no grupo de apoio, a experiência de muitos deles enfrentando situações de preconceito na sala de aula e constatações que levam a conclusão de que o projeto de educação só será efetivo se houver a participação de toda comunidade escolar.

Dos professores orientadores que responderam o questionário avaliativo $18.75 \%$ são da área de geografia, $12.50 \%$ de pedagogia, $12.50 \%$ de gestão escolar, $12.50 \%$ de história, $12.50 \%$ de língua inglesa, $12.50 \%$ de língua portuguesa, $6.25 \%$ de educação física, $6.25 \%$ de artes (educação artística) e $6.25 \%$ de química.

Os professores orientadores manifestaram que as atividades colaborativas e formativas com os colegas docentes da rede de ensino básico foram eficientes para inferir as mudanças ocasionadas pelo percurso do PDE na atividade profissional do professor. Recomendam que mantenham o projeto que iniciaram no PDE na sua escola como estratégia de manejo de situações identificadas como problemáticas.

Artigos escritos após o professor PDE ter finalizado sua participação oficial no programa publicados em revista na área da educação comprovam que alguns professores adquiriram o gosto pelo trabalho acadêmico.

Dos coordenadores pedagógicos do PDE/UFPR, 75\% responderam o questionário avaliativo.

WALFLOR, Marlene F. G. M; DEVAI, Orlando C.; VIEIRA, Geraldine M.R. Interação interinstitucional: o caso do Programa de Desenvolvimento da Educação (PDE) / Universidade Federal do Paraná (UFPR) e o desenvolvimento regional. Extensão em Foco, Curitiba: Editora da UFPR, nr.9, jan/jun 2014, p.61-78. ISSN 2358-7180. 
Os coordenadores manifestaram que a alocação do PDE/UFPR na PróReitoria de Extensão e Cultura e Setores da UFPR com destinação de espaço físico, liberação de profissionais docentes e administrativos para compor a equipe do PDE/UFPR e destinação de bolsas para alunos da graduação participarem das atividades junto a equipe do PDE/UFPR foi significativa para a realização do programa de extensão. Expressaram que a gestão pedagógica do PDE/UFPR integrada com o ensino, pesquisa e extensão possibilitou: ao professor PDE cursar disciplinas de diferentes cursos de graduação e pósgraduação; a inserção do projeto do professor PDE na área temática do projeto do professor orientador e a inserção do professor PDE nas diferentes ações de extensão realizadas na UFPR e que o retorno dos professores da rede pública à universidade, bastante enriquecedor, gerou oportunidades de atualização do fazer profissional do professor, contribuiu para a análise reflexiva e ajustes no trabalho desenvolvido nas escolas. Salientaram que artigos produzidos a partir dos projetos realizados nas escolas se constituíram fontes de consulta para outros professores da rede pública e para alunos da UFPR.

Destacam que 0 PDE/UFPR viabiliza a oferta de atividades diversificadas para professores PDE com o estabelecimento de parcerias com outras Instituições de Ensino Superior (IES) federal e estadual, participantes do PDE/PR e que existem perspectivas de parcerias futuras entre a universidade e escolas da rede pública estadual. Acreditam que o PDE/UFPR se consolida, tanto como política de articulação com diferentes setores e departamentos da universidade, quanto com a educação básica.

Os resultados da avaliação da comparação dos parâmetros do Marco Lógico em relação aos participantes e os resultados obtidos após a conclusão das três turmas do PDE/UFPR mostram que dos 552 professores PDE selecionados pelo PDE e encaminhados para a UFPR, 546 concluíram o PDE/UFPR registrando frequência superior a $80 \%$ nas atividades de aprofundamento teórico (ensino, pesquisa e extensão) e 100\% de aproveitamento nas atividades de integração teórico-práticas (projeto de intervenção pedagógica na escola, produção didático-pedagógica e artigo científico). Não foram registrados casos de evasão, havendo apenas casos

WALFLOR, Marlene F. G. M; DEVAI, Orlando C.; VIEIRA, Geraldine M.R. Interação interinstitucional: o caso do Programa de Desenvolvimento da Educação (PDE) / Universidade Federal do Paraná (UFPR) e o desenvolvimento regional. Extensão em Foco, Curitiba: Editora da UFPR, nr.9, jan/jun 2014, p.61-78. ISSN 2358-7180. 
isolados de desligamento de professor PDE na turma de 2008/2009, em que ocorreu um caso de desistência por motivo de saúde e um caso de ausente desde o início do Programa e na turma 2009/2011, em que ocorreu um caso de aposentadoria compulsória, dois casos de desligamento voluntário por motivos pessoais e um caso de processo de exclusão de professor.

Outro ponto a salientar é o posicionamento dos professores PDE e professores orientadores que responderam ao questionário. Estes revelam que a formação continuada proporcionou uma possibilidade de amadurecimento e reflexão coletiva para todos os segmentos envolvidos. Segundo depoimento dos professores constata-se como resultados: a construção do conhecimento relacionado às realidades das escolas e o consequente aumento da autoestima de pessoas e comunidades (em especial dos professores participantes); a riqueza de troca de experiências, em termos e modos de abordagem ou materiais utilizados em salas de aula; o desenvolvimento do espírito cooperativo e o despertar da consciência do caráter dinâmico e interativo de nossas concepções e práticas profissionais; o aprendizado acerca de práticas pedagógicas; a vivência e a importância do espírito de liderança dentro do grupo de trabalho para torná-lo eficaz. Outro resultado relevante é na formação inicial dos estudantes de cursos licenciaturas da UFPR que participaram no desenvolvimento das atividades realizadas dentro do programa (em especial nos grupos de estudos) e a abertura de oportunidades de estágio, para esses alunos, em escolas da rede pública onde atuam professores PDE.

De outro lado constata-se que o programa, procurou em todos os momentos garantir espaços de expressão para os participantes, evitando uma estrutura monovocal, onde só a universidade sabe e pode dizer.

As entrevistas nas escolas permitiram observar que o professor, ao vivenciar o processo de formação continuada, está aperfeiçoando sua função de educar, no sentido de refletir e agir sobre o seu trabalho. Entendendo a escola como local de convivência de experiências múltiplas, consequentemente, ela representa, para a formação continuada, um espaço de construção de conhecimentos da prática docente.

WALFLOR, Marlene F. G. M; DEVAI, Orlando C.; VIEIRA, Geraldine M.R. Interação interinstitucional: o caso do Programa de Desenvolvimento da Educação (PDE) / Universidade Federal do Paraná (UFPR) e o desenvolvimento regional. Extensão em Foco, Curitiba: Editora da UFPR, nr.9, jan/jun 2014, p.61-78. ISSN 2358-7180. 
Ao analisar o programa PDE não é difícil verificar os benefícios individuais aos professores participantes do programa. $O$ retorno individual é bastante evidente, mas o social nem sempre é considerado. O ganho social a partir de um efeito multiplicador tende a ser superior ao individual, pois os seus resultados extrapolam os benefícios do indivíduo que se qualifica, principalmente quando consideramos ambientes de aglomeração humana como na escola e em espaços urbanos onde ocorre intensa troca de informação.

O fato de o indivíduo qualificado repassar seu conhecimento para a sociedade, além de gerar benefícios à coletividade faz com que este conhecimento permaneça mesmo após sua saída. Com o aumento do conhecimento assimilado pela sociedade, a própria universidade ganha em qualidade, quando recebe alunos, professores, pesquisadores, e técnicos com melhor preparo. Esta acumulação de conhecimento social leva a um desenvolvimento regional sustentado.

Com o desenvolvimento da sociedade pela acumulação do conhecimento são criadas vantagens comparativas em relação às outras regiões, atraindo talentos e novos investimentos intensivos em tecnologia, aumentando produtividade e a renda per capta, o que proporciona melhoria da qualidade de vida da população como objetivo fim. $\mathrm{O}$ aumento da renda eleva $\mathrm{o}$ consumo e o investimento produtivo, refletindo também num maior fluxo de recursos para programas educacionais, resultando em um círculo virtuoso sustentado, dado que há sincronia entre a formação e na utilização de profissionais qualificados, tanto na área educacional quando na área produtiva de alta tecnologia.

A extensão universitária na qual o PDE se destaca vem no sentido de encurtar o caminho entre o conhecimento teórico e o conhecimento prático dentro da lógica da educação continuada, sendo que a soma ou interação dos conhecimentos produz resultados superiores às duas partes, quando comparados com a gestão de forma separada.

Sem a extensão e a pesquisa, a universidade perderia significativamente sua importância na melhoria da qualidade de vida da

WALFLOR, Marlene F. G. M; DEVAI, Orlando C.; VIEIRA, Geraldine M.R. Interação interinstitucional: o caso do Programa de Desenvolvimento da Educação (PDE) / Universidade Federal do Paraná (UFPR) e o desenvolvimento regional. Extensão em Foco, Curitiba: Editora da UFPR, nr.9, jan/jun 2014, p.61-78. ISSN 2358-7180. 
população, limitando-se ao papel de mera reprodutora de conhecimento teórico. Este conhecimento teórico gestado em outras realidades, nem sempre é compatível ou aplicável às comunidades em que está inserido. O bom relacionamento entre a universidade e a sociedade e demais órgãos governamentais é de grande importância para o seu desenvolvimento, dado o aprendizado resultante do envolvimento do professor universitário com a realidade do profissional egresso da própria universidade e de outras instituições com experiências e formações diferenciadas, permitindo a reavaliação de sua didática e conteúdos e mesmo a possibilidade de comparálo com o de outras instituições no momento que este egresso faz a aplicação do conhecimento adquirido na universidade.

\section{Conclusão}

A implementação do Programa de Extensão Universitária "Desenvolvimento da Educação - PDE/UFPR", evidenciou que as instituições universitárias são importantes para a formação continuada de profissionais da educação por possuírem um relevante sistema de recursos a serviço dos projetos de escolas e servirem como base de apoio e inovações.

Por meio do programa, espera-se consolidar a relação da Universidade com a Escola e facilitar o necessário diálogo com os agentes educacionais da região. Acredita-se, ainda, que esta ação de extensão possibilitará aos alunos da UFPR a oportunidade de dialogar com os educadores da região e assim conhecer e discutir sobre suas práticas, a escola e o processo educativo, por meio de elaboração de atividades a serem oferecidas e da difusão de materiais de apoio teórico. O trabalho conjunto - Universidade e Escola - propiciará um incremento à pesquisa (de docentes e alunos), o aperfeiçoamento das ações de extensão e a difusão de novas ideias através de artigos, e monografias de graduação e pós-graduação, contribuindo desta forma, cada vez mais para o desenvolvimento regional.

WALFLOR, Marlene F. G. M; DEVAI, Orlando C.; VIEIRA, Geraldine M.R. Interação interinstitucional: o caso do Programa de Desenvolvimento da Educação (PDE) / Universidade Federal do Paraná (UFPR) e o desenvolvimento regional. Extensão em Foco, Curitiba: Editora da UFPR, nr.9, jan/jun 2014, p.61-78. ISSN 2358-7180. 


\section{REFERÊNCIAS}

AMBROSETTI, N. B.; RIBEIRO, M. T. M. Universidade e formação continuada de professores: algumas reflexões. Anais da $28^{a}$ reunião da ANPEd. ANPEd: Caxambu-MG, 2005 Disponível em http:// 28reuniao.anped.org.br Acesso em 28 de junho de 2010.

BACIGALUPO, L. E. Universidad y Desarrollo Regional: una aproximación desde los conceptos de Sociedad Civil y Responsabilidad Social Universitária. Entrevista. DC, 2004. Disponível em: <www.pucp.edu.pe>. Acesso em: maio 2009.

BANCO INTERAMERICANO DE DESARROLLO - BID. Oficina de Evaluación. Evaluación: uma herramenta de gestión para mejorar el desempeño de los poyetes (Marco Lógico). DC, 1997. Disponível em: <www.ladb.org/ont/evo/SPBook.Htm>. Acesso em: 15 de maio 2009.

BRASIL. Ministério da Educação. Secretaria de Educação Superior. Plano Nacional de Extensão Universitária. Fórum de Pró-Reitores de Extensão das Universidades Públicas Brasileiras. Brasília - DF, 2001.

. Ministério da Educação. Decreto 6.755, de 29 de janeiro de 2009. Institui a Política Nacional de Formação de Profissionais do Magistério da Educação Básica, disciplina a atuação da Coordenação de Aperfeiçoamento de Pessoal de Nível Superior, CAPES no fomento a programas de formação inicial e continuada, e dá outras providências. Diário Oficial da União, Brasília - DF, 30 jan. 2009.

DICHIKSSON, A. Universidad, Sociedad del Conocimiento x Nueva Economia. UNESCO. Venezuela, 2006.

FREITAS, H. C. L. A reforma do ensino superior no campo da formação dos profissionais da educação básica: As políticas educacionais e movimento dos educadores. Educação \& Sociedade, n. 68, dez. 1999.

GIBBONS, M. Pertinência de la educación superior em el siglo XXI. Banco Mundial, 1998.

PARANÁ. Lei Complementar no 130, de 14 de julho de 2010. Regulamenta o Programa de Desenvolvimento Educacional - PDE, instituído pela Lei Complementar № 103/2004. Diário Oficial da República Federativa do Brasil, Brasília - DF, n. 8.266, jul. 2010.

. Secretaria de Estado da Educação. Programa de Desenvolvimento Educacional: documento - síntese. SEED. Curitiba, 2007.

WALFLOR, Marlene F. G. M; DEVAI, Orlando C.; VIEIRA, Geraldine M.R. Interação interinstitucional: o caso do Programa de Desenvolvimento da Educação (PDE) / Universidade Federal do Paraná (UFPR) e o desenvolvimento regional. Extensão em Foco, Curitiba: Editora da UFPR, nr.9, jan/jun 2014, p.61-78. ISSN 2358-7180. 